\title{
Morphological and molecular characterization of Paragonimus species isolated from freshwater crabs in Yunnan, China.
}

\section{Qiu-Hong Shu}

The Second Affiliated Hospital of Kunming Medical University

\section{Shu-De Li}

School of Basic Medical Sciences, Kunming Medical University

\section{Ming Tian}

School of Basic Medical Sciences, Kunming Medical University

\section{Wen-Wei Bai}

The Second Affiliated Hospital of Kunming Medical University

\section{Yong Meng}

The Second Affiliated Hospital of kunming Medical University

\section{Shu-Mei-Qi He}

School of Basic Medical Sciences, Kunming Medical University

Hui Su

School of Basic Medical Sciences, Kunming Medical University

\section{Miao-Miao Wang}

School of Basic Medical Sciences, Kunming Medical University

Wenlin Wang ( $\square$ wenliwang321@gmail.com )

Faculty of Basic Medicine, Kunming Medical University

\section{Research}

Keywords: Paragonimus; fresh water crabs; metacercariae; phylogenetic analysis

Posted Date: May 13th, 2020

DOl: https://doi.org/10.21203/rs.3.rs-27578/v1

License: (c) (i) This work is licensed under a Creative Commons Attribution 4.0 International License. Read Full License 


\section{Abstract \\ Background}

Paragonimus species are highly prevalent in ethnic minority areas of Yunnan, China. The objective of the study is to isolate and identify Paragonimus from natural habitat and compare the phylogenetic diversity of Paragonimus in Yunnan province, China.

\section{Methods}

Metacercariae of Paragonimus was isolated from crabs and morphologic identification was performed by microscopy. Metacercariae were injected into experimental Paragonimus free Sprague Dawley rats. After 112 days, adult worms and eggs were isolated from multiple organs. Morphologic identification confirmed the initial identification. DNA was extracted from the adult worms and molecular characterization was performed by amplification and sequencing of CO1 and ITS2 regions followed by phylogenetic analysis.

\section{Results}

Out of 447 crabs captured, 186 crabs were found to be infected. A total of 5 occurrences of Paragonimus was observed from naturally infected crabs. Paragonimus microrchis (2), P,heterotremus (1), P.proliferus (1) and P.skrjabini (1) were isolated and identified. A total of 32 sequences downloaded from national center for biotechnology information and 5 sequences generated in the study was used for phylogenetic analysis. Phylogenetic analysis revealed robustness of the double loci method as against the single locus method with either CO1 or ITS2 alone. Considerable phylogenetic diversity depending on the geographic location was observed.

\section{Conclusion}

Paragonimus species isolated from Yunnan province, China were phylogenetically diverse and the analysis revealed the clustering of multiple species of Paragonimus isolated from different geographic locations.

\section{Background}

Trematodes belonging to the genus Paragonimus are important parasites causing zoonotic infections in vertebrates including human beings. They are of socio-economic importance since the route of infection is mainly food borne (1). The life cycle of Paragonimus is relatively complex that requires a minimum of three hosts including a definitive host and two intermediate hosts (2). The first and second intermediate hosts are frequently snails belonging to the families Assimineidae and Hydrobiidae and crabs belonging 
to the families Potamidae and Parathelphusidae (3). Different species of Paragonimus have their own predilection for infecting specific genera of snails and crabs and hence the epidemiological prevalence of different species of Paragonimus is determined by the existence of suitable hosts. Some species of Paragonimus have been identified only from intermediate hosts in certain geographies which suggests infection and maintenance in non-human mammals $(4,5)$.

The genus Paragonimus is a genus rich in species diversity that has been reported from varied geographies including Asia, Africa, Central and South America spanning both tropical and temperate climates (6). The species diversity is also reflected on the varied phylogenies of Paragonimus based on which it could be divided into 3 species complexes. Paragonimus westermani complex is the most commonly reported species complex that has been reported from south and south east Asia(7). Paragonimus heterotremus species has been predominantly isolated and reported from South East Asia and Paragonimus skrjabini complex has been reported from China and East Asia $(6,8,9)$. The allelic diversity and ontogenetic changes have been previously studied with isozymes which has been superseded with molecular approaches(10). There were previous instances of discordance in morphology and the ontogenetic changes predicted by both molecular and isozyme-based methods(11). One of the main reasons for the observed discordance is the lack of clarity on the species boundaries and also mendelian polymorphism leading to morphological changes causing creation of distinct species that are interfertile(12).

DNA sequence data can be used to construct phylogenetic trees that can be used to infer evolutionary trends including insights into speciation and geographic spread(13). Further, the morphological differences in adult and metacercariae that could be used to differentiate different species is also limited. This expands the potential role of DNA sequence analysis to assist in confirming species distribution and phylogenetic diversity. Currently, nuclear (Internal transcribed spacer (ITS) 2) and mitochondrial DNA (cytochrome c oxidase subunit 1 (CO1)) sequences are used for assessing the phylo-geographies(14).

Nearly $80 \%$ of the Paragonimus species reported worldwide were from China which also has the highest disease burden in the world (15). Due to the high disease burden and eating habits, China represents an ecological hotspot for the dissemination and further evolution of Paragonimus species. Hence frequent phylogenetic analysis of Paragonimus species from non-mammalian hosts is required to keep a check on paragonimiasis and also to suggest suitable life-style modifications for populations at risk for acquiring paragonimiasis. Hence, in this study, we report the phylogenetic diversity and evolutionary relationship of Paragonimus species isolated from crabs in Yunnan province, China.

\section{Methods}

\section{Parasitological methods}

The Paragonimus metacercariae were isolated from naturally infected mountain crabs belonging to the genus Potamon, the second intermediate hosts, from Tongchang Town, Jinping County, Yunnan Province, China. The habitat included fast moving streams with no adjacent vegetation. The identification of the 
secondary hosts was done according to the classification method of "Chinese Medical Crustaceans"(16). The crabs were smashed in a mortar followed by sieving and washed with distilled water into a sedimentation cup with distilled water. The filtration was done with a filter of pore size 200 microns and 1000 microns. The supernatant was discarded after 20 minutes and the same step was repeated four to five times. The sediment was then placed in a glass dish for microscopic biological observation. The metacercariae of Paragonimus were counted under the microscope and a part of the sediment with the metacercariae were fixed with absolute ethanol and stored in a refrigerator at $4 \mathbb{} \mathrm{C}$ for molecular biological experiments.

\section{Experimental infection}

Freshly isolated, live metacercariae were then injected intraperitoneally (15 metacercariae per rat) into paragonimiasis-negative Sprague Dawley (SD) rats (purchased from the Laboratory Animal Department of Kunming Medical University). All the animals were handled in accordance with the Guide for the Care and Use of Laboratory Animals published by the US National Institutes of Health (NIH Publication No. 8523, revised 1985). All experimental protocols were approved by the Animal Care and Use Committee of Kunming Medical University (reference: KMMU2015002). Experimental animal inoculation was performed based on the sampling point. After subcutaneous injection, five SD rats were sacrificed on second, fourth and sixth week to confirm infection of rats with cercariae of Paragonimus. After 112 days of injection, the SD rats were dissected to isolate the cysts, eggs and adult worms from the muscles, abdomen, liver, thoracic cavity and lungs of SD rats. The isolated adult worms were used for genomic DNA extraction and for preparing permanent slides for microscopic confirmation by fixing them on to glass slides with alcohol, formalin and acetic acid.

\section{Microscopic identification}

The different characteristic features like the shape, body size, and measurement and position of the suckers were used for microscopic and morphologic characterization.

\section{Molecular analysis}

Genomic DNA was extracted from both the adult worms from SD rats and also from the metacercariae extracted from crab using QIAamp DNA Mini kit (QIAGEN, Hilden, Germany). The whole process was carried out in strict accordance with the instructions of the manufacturer. The final elution of DNA was done with $100 \mu \mathrm{L}$ of distilled water. The extracted total genomic DNA was quantified and stored in the refrigerator at $-20 \otimes C$ until further use.

\section{Polymerase chain reaction amplification of $\mathrm{CO} 1$ and ITS2}

Polymerase chain reaction (PCR) was performed with primers targeting a fragment of the $\mathrm{CO} 1 \mathrm{gene}$ and the ITS2 region synthesized by Shanghai Bioengineering Co., Ltd. The primers used for amplifying COI gene fragments were COIF- 5'GAGGTGTATGTCCTGATTTTGCC-3' and CO1R5'GACCTCACCCAATGACCCTGCAACA3' and the primers for amplifying ITS2 gene fragments were ITS2F- 
5'GGGTACCGGATCACTCGCTCGGTG3' and ITS2R-5'GGGGATCCTGGTTGCCTTAGTCTCCGC3'. PCR was performed in $25 \mu \mathrm{L}$ volume with $2 \mu \mathrm{L}$ of template DNA corresponding to $0.1 \mathrm{ng}$ and $1 \mu \mathrm{L}$ of primers (10 $\mu \mathrm{mol} / \mu \mathrm{L}$ ), $2.5 \mu \mathrm{L}$ of $10 X$ PCR buffer, $1 \mu \mathrm{L}$ of $10 \mathrm{mM}$ dNTP, $0.1 \mu \mathrm{L}$ ( 0.5 units) of Taq enzyme ( $5 \mathrm{U} / \mathrm{ul}$ ) and $17.4 \mu \mathrm{L}$ of PCR grade water. The setting up of PCR reaction was done in ice bath. The PCR amplification was conducted in TaKaRa PCR instrument (Bao Biology Co., Ltd.) and the amplification conditions were as follows: initial denaturation of $95 \rrbracket \mathrm{C}$ for 3 minutes followed by 35 cycles of denaturation at $93 \rrbracket \mathrm{C}$ for 1

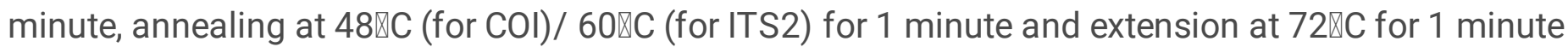
followed by final extension at $72 \varangle \mathrm{C}$ for 5 minutes. The expected length of the PCR fragments was 500750 base pairs. Detection of PCR amplified products was done by agarose gel electrophoresis with 1.5\% agarose gel immersed in 1.0\% Tris-Acetate-EDTA buffer stained with ethidium bromide. The purity and quantity were estimated by imaging the gel in gel documentation system (Bio-Rad company).

The PCR products were then subjected to bidirectional sequencing using the same PCR primers by Shanghai Biotechnology Co., Ltd (Hitachi fluorescent DNA sequencer SQ-3000). The forward and reverse sequences were then manually curated and aligned with the Dnastar v7.1 software and the consensus sequence were used for bio-informatic analysis.

The initial quality check of the sequences was done by checking the coverage and alignment with previously submitted sequences in National Center for Biotechnology Information (NCBI) using the BLAST tool. Previously submitted sequences of COI and ITS2 were retrieved from NCBI and compared with the sequences obtained in this study with ClustalX software with default parameters. Phylogenetic analysis was done by the Maximum parsimony Likelihood (ML) method as per the Kimura 2-parameter model. Nearest-Neighbor-Interchange (NNI) was used for the ML Heuristic Method with bootstrap replicates of 1000 . The analysis was performed with MEGA5.0 software (14). The cut-off value for consensus tree was set to $75 \%$.

\section{Results}

\section{Morphological identification}

\section{Metacercariae}

A total of 447 crabs captured from stream in Tongchang Town, Jinping County, Yunnan Province, China, were included for this study. Out of 447 crabs 186 crabs were found to be infected. A total of 551 metacercariae were isolated and identified from the 186 crabs (2.96 per crab). From each crab 4 to 98 metacercariae were obtained with an average of 22. The size of the metacercariae was found to be $0.419 \mathrm{~mm} \times 0.398 \mathrm{~mm}$. The average thickness of the outer wall and inner wall were found to be $0.004 \mathrm{~mm}$ and $0.012 \mathrm{~mm}$, respectively, using microscopic technique. Further, the larvae were surrounded by two larger excretory cysts and intestinal branches.

\section{Adult worm}


After experimental infection, from each SD rat 2 to 4 adult worms were retrieved (Fig 1). Adult worms were distributed in both lungs and muscles, but seldom found in liver and brain of affected SD rats. A total of 5 adult flukes recovered from SD rats were used for morphological and molecular analysis. The average size of the gravid adult worms was $6.7 \mathrm{~mm} * 3.8 \mathrm{~mm}$. The tegmental spines and were mainly clustered into clusters of 4-6 around the ventral sucker. The spines around the oral sucker were short, small, and solitary. The size of the oral and ventral suckers was $0.43 \mathrm{~mm} * 0.53 \mathrm{~mm}$ and $0.75 \mathrm{~mm}$ * $0.79 \mathrm{~mm}$, respectively. The reproductive organs were observed as large uterine masses, with size of $2.695 \mathrm{~mm}$ * $2.107 \mathrm{~mm}$. The testicle is in the inferior half of the worm with dimensions of $1.72 \mathrm{~mm}$ * $1.38 \mathrm{~mm}$.

Morphological identification of the metacercariae isolated from the infected crabs and adult worms from the SD rats led to the identification of 4 species of Paragonimus, namely, $P$. proliferus, $P$. microrchis, $P$. heterotremus and P. skrjabini.

\section{Molecular identification}

PCR amplification of the CO1 and ITS2 regions, followed by agarose gel electrophoresis, revealed amplicons of about 500 and $750 \mathrm{bp}$ in length, respectively. BLAST searches using our sequences as queries found matches with 100\% coverage in GenBank. Further, only the C01 sequence of Paragonimus microrchis differed slightly from previously published sequences. All the other sequences obtained in this study revealed $100 \%$ identity to previously published sequences.

\section{Phylogenetic analysis}

A total of 33 gene sequences were downloaded from NCBI for this analysis (table 1). In the phylogenetic tree of COI gene, Paragonimus proliferus, Paragonimus heterotremus, Paragonimus skrjabini were clustered with the same species, and the confidence values of their branches were more than $95 \%$ (except for the support degree of the branches where sample 30 was located was $88 \%$ ) (figure 2). Paragonimus microrchis clustered with Paragonimus bangkokensis. Congruent phylogenetic relationship were observed with ITS2 phylogenetic tree (figure 3) In the phylogenetic tree constructed with the combined dataset (figure 4) of $\mathrm{COI}$ and ITS2 datasets, Paragonimus proliferus, Paragonimus heterotremus and Paragonimus skrjabini still clustered with the same species, and their branch confidence values were more than 94\%. Paragonimus microrchis remained clustered with Paragonimus bangkokensi. 


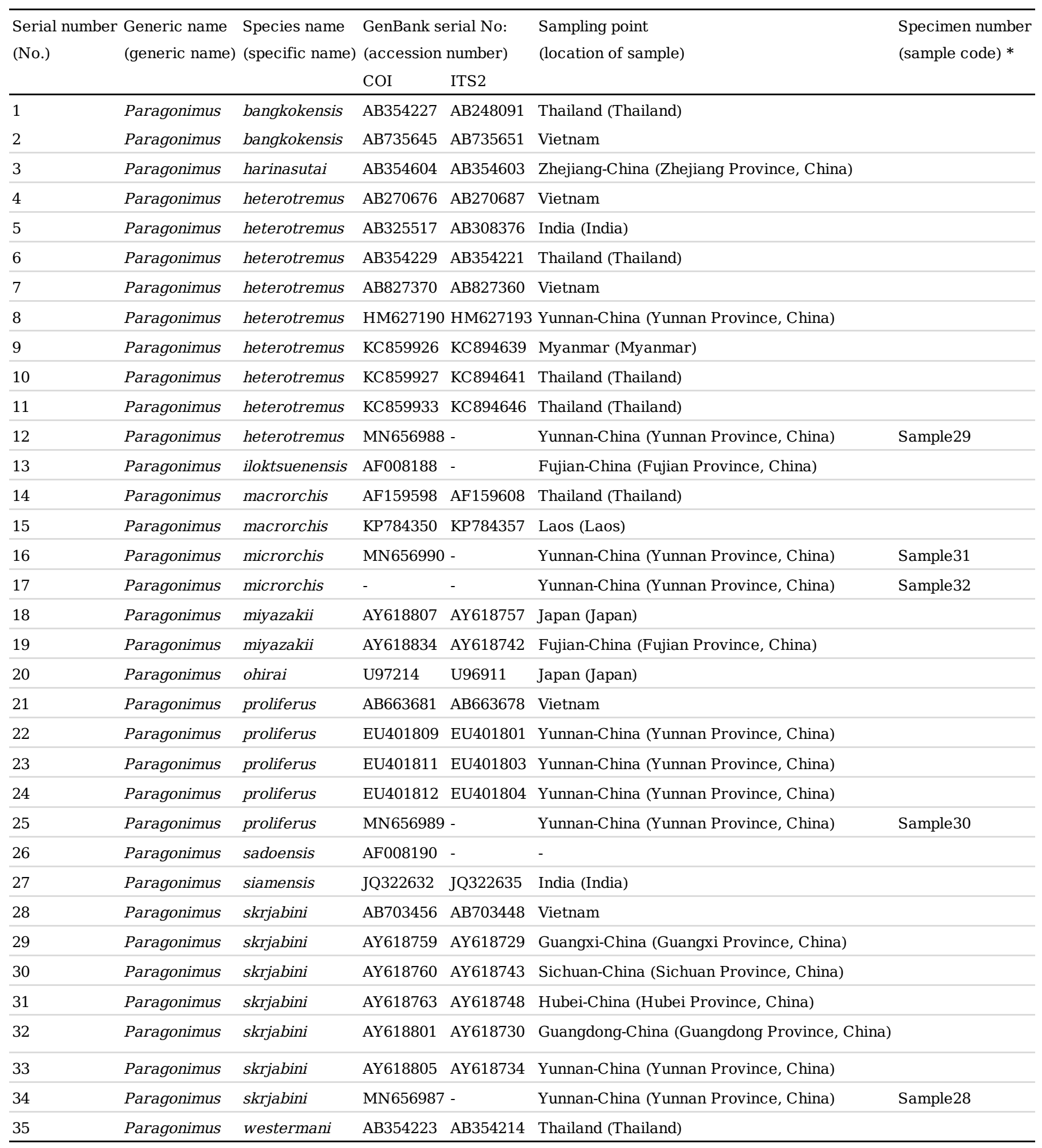

\section{Discussion}

China is known for the endemic diversity of Paragonimus species wherein 38 out of the 50 globally reported species have been identified $(15,17)$. Hence, China is a hotspot for phylogenetic diversity among the previously reported species. The probability of phylogenetic diversification is more in naturally infected secondary hosts like crabs, wherein other ecological factors may drive diversification (18). 
Hence, we determined the occurrence and phylogenetic diversity of Paragonimus species in Yunnan province China. In the current study, out of the sequenced samples, 2 were P.microrchis and 1 each of P.heterotremus, P. skrjabini and P.proliferus.

Since the differential identification features of cercariae are not well marked, most often the species identification is done at the metacercariae stage from the secondary intermediate hosts (3). In this study morphological identification made at the metacercariae stage and adult stage were concordant with each other further substantiating the role of morphological identification. This was also confirmed with PCR amplification and sequencing of $\mathrm{CO} 1$ and ITS2 region.

Phylogenetic analysis with previously published sequences and the sequences generated in this study revealed concordance between the single locus (CO1 or ITS2) and double loci (CO1 and ITS2) phylogenetic analysis. The phylogenetic analysis revealed the presence of 3 distinct clusters of Paragonimus consisting of P.siemensis, $P$. westermani in cluster $1, P$. skrjabini, P. miyazakii, P. proliferus, $P$. macrorchis and $P$. heterotremus in cluster 2 and $P$. microrchis, $P$. bangkokensis, $P$. harinasutai, $P$. iloktsuensis and $P$. sadoensis in cluster 3 . Extensive branching was observed in cluster 2 wherein P.skrjabini isolated from different regions in China showed as distinct branches. Further, P.heterotremus clustered into a stable branch with $P$. heterotremus from south east Asia clustering into a single monophyletic branch group distinct from the P.heterotremus from India. Similarly, P.proliferus also clustered as a monophyletic group in most cases (except in NJ trees constructed by ITS2). The relationship between $P$. macrorchis and the first two branches is not clear, but in the MP phylogenetic tree of ITS2 data sets, COI and ITS2 combined data sets, P.heterotremus tends to be clustered into one branch.

By comparing the results of constructing adjacent NJ and MP phylogenetic trees from COI, ITS2, $\mathrm{COI}$ and ITS2 datasets, it is obvious that different data sets and tree-building methods have great influence on the robustness of phylogenetic trees. In phylogenetic trees constructed from single gene datasets, the number of branches with more than $75 \%$ support is less than that constructed from joint datasets, especially self-exhibition, which deserves significant improvement. The phylogenetic tree constructed by MP method using COI and ITS2 data sets has a high robustness, and P.macrorchis and P.heterotremus were clustered together, which is supported by $91 \%$.

Among the Paragonimus spp. reported in this study, all the species were previously reported from China. A study by Lou et al., utilized similar approaches in identifying the phylogenetic diversity of Paragonimus in China and reported clustering of isolates based on geographic location which was in accordance to the current study(14). P.skrjabini was previously reported from Gansu, Shanxi, Yunnan, Guangxi, Guizhou, Sichuan and Jiangxi provinces of China. While, $P$ heterotremus has been reported only from Yunnan and Guangxi provinces(2).

Phylogenetic analysis has varied values in different organisms. In Paragonimus, where there is no consensus on speciation with both morphological and molecular characteristics, phylogenetic analysis 
helps in determining the phylogeographies(7). The results of our study further substantiated the conservative phylogeographies revealed through analysis of Cox 1 and ITS 2 regions.

The study is not without limitations. The study sites were localized to Yunnan province in China; hence the results may not be extended to other parts of China. Secondly, we used maximum parsimony method which is infrequently used despite the robustness mainly because of the nature of assumptions deployed in the method. Nevertheless, maximum likelihood method which was also used in other studies also has drawbacks.

In conclusion, a total of 4 morphologically different species of Paragonimus was isolated from Yunnan province, China. Phylogenetic analysis using the polymorphisms in two different loci revealed considerable variations in the species of Paragonimus identified in different geographic locations with clustering of Paragonimus based on geographic location.

\section{Conclusions}

Paragonimus species isolated from Yunnan province, China were phylogenetically diverse and the analysis revealed the clustering of multiple species of Paragonimus isolated from different geographic locations

\section{Abbreviations}

DNA

Deoxyribonucleic acid

ITS

Internal Transcribed Spacer

C01

Cytochrome C Oxidase Subunit 1

SD

Sprague Dawley

PCR

Polymerase chain reaction

EDTA

Ethylenediaminetetraacetic acid

NCBI

National Centre for Biotechnology Information

$\mathrm{ML}$

Maximum Parsimony likelihood

$\mathrm{NNI}$

Nearest-Neighbor-Interchange 


\section{Declarations}

Ethics approval and consent to participate: Not applicable

Consent for publication: Not applicable

Availability of data and materials: The datasets used and/or analysed during the current study are available from the corresponding author on reasonable request.

Competing interests: The authors declare that they have no competing interests.

Funding: The study was funded by Yunnan Science and Technology Department - Kunming Medical University Applied Basic Research Joint Major Project, Mechanism research of gentiopicroside in the treatment of pulmonary damage caused by Paragonimus proliferus (Grant number-201801PH00065).

Author's Contributions: Qiu-Hong Shu, Shu-De Li, Ming Tian, Wen-Wei Bai and Yong Meng contributed substantially to study conception and design, acquisition, analysis and interpretation of data, revised the final version of the manuscript. Shu-Mei-Qi He, Hui Su, Miao-Miao Wang and Wenlin-Wang contributed substantially to study conception and design, acquisition, analysis and interpretation of data. All authors read and approved the final version to be published.

\section{References}

1. Singh TS, Mutum SS, Razaque MA. Pulmonary paragonimiasis: clinical features, diagnosis and treatment of 39 cases in Manipur. Trans R Soc Trop Med Hyg. 1986;80(6):967-71.

2. Yoshida A, Doanh PN, Maruyama H. Paragonimus and paragonimiasis in Asia: An update. Acta Trop. 2019 Nov; 1:199:105074.

3. Blair D, Xu ZB, Agatsuma T. Paragonimiasis and the genus Paragonimus. Adv Parasitol. 1999;42:113-222.

4. Doanh NP, Tu AL, Bui TD, Loan TH, Nonaka N, Horii Y, et al. Molecular and morphological variation of Paragonimus westermani in Vietnam with records of new second intermediate crab hosts and a new locality in a northern province. Parasitology. 2016;143(12):1639-46.

5. Song J-H, Dai F, Bai X, Kim T-I, Yang H-J, Kim T-S, et al. Recent Incidence of Paragonimus westermani Metacercariae in Freshwater Crayfish, Cambaroides similis, from Two Enzootic Sites in Jeollanamdo, Korea. Korean J Parasitol. 2017 Jun;55(3):347-50.

6. Blair D, Nawa Y, Mitreva M, Doanh PN. Gene diversity and genetic variation in lung flukes (genus Paragonimus). Trans R Soc Trop Med Hyg. 2016 Jan;110(1):6-12.

7. Blair D. Genomes of Paragonimus westermani and related species: current state of knowledge. Int J Parasitol. 2000 Apr 10;30(4):421-6.

8. Blair D, Chang Z, Chen M, Cui A, Wu B, Agatsuma T, et al. Paragonimus skrjabini Chen, 1959 (Digenea: Paragonimidae) and related species in eastern Asia: a combined molecular and 
morphological approach to identification and taxonomy. Syst Parasitol. 2005 Jan;60(1):1-21.

9. Sanpool O, Intapan PM, Thanchomnang T, Janwan P, Nawa Y, Blair D, et al. Molecular Variation in the Paragonimus heterotremus Complex in Thailand and Myanmar. Korean J Parasitol. 2013 Dec;51(6):677-81.

10. Blair D. Molecular variation in fasciolids and Paragonimus. Acta Tropica. 1993 May 1;53(3):277-89.

11. Agatsuma T, Habe S. Genetic variability and differentiation of natural populations in three Japanese lung flukes, Paragonimus ohirai, Paragonimus iloktsuenensis and Paragonimus sadoensis (Digenea: Troglotrematidae). J Parasitol. 1986 Jun;72(3):417-33.

12. Habe S, Agatsuma T, Hirai H. Evidence for metacercarial polymorphism in lung flukes, Paragonimus ohirai and Paragonimus iloktsuenensis. J Parasitol. 1985 Dec;71(6):820-7.

13. Blair D, Wu B, Chang ZS, Gong X, Agatsuma T, Zhang YN, et al. A molecular perspective on the genera Paragonimus Braun, Euparagonimus Chen and Pagumogonimus Chen. J Helminthol. 1999 Dec;73(4):295-9.

14. Lou HQ, Hu Y, Zhang L, Zhang YJ, Tu PG, Yu XT, et al. Epidemiological survey and phylogenetic analysis of Paragonimus westermani isolates in Jinhua, China. African Journal of Biotechnology. 2011 Jan 1;10(65):14286-92-14292.

15. Liu Q, Wei F, Liu W, Yang S, Zhang X. Paragonimiasis: an important food-borne zoonosis in China. Trends Parasitol. 2008 Jul;24(7):318-23.

16. Nawa Y, Thaenkham U, Doanh PN, Blair D. Helminth-Trematode: Paragonimus westermani and Paragonimus Species. In: Motarjemi Y, editor. Encyclopedia of Food Safety [Internet]. Waltham: Academic Press; 2014 [cited 2020 Jan 16]. p. 179-88. Available from: http://www.sciencedirect.com/science/article/pii/B9780123786128001591.

17. Zhang $X$, Wang Y, Wang G, Chen W, He X, Niu H, et al. Distribution and clinical features of Paragonimiasis skrjabini in Three Gorges Reservoir Region. Parasitol Int. 2012 Dec;61(4):645-9.

18. Rios RS, Salgado-Luarte C, Gianoli E. Species Divergence and Phylogenetic Variation of Ecophysiological Traits in Lianas and Trees. PLOS ONE. 2014 Jun;10(6):e99871. 9(.

\section{Figures}

\section{Figure 1}

Paragonimus cysts with adult worms isolated from SD rats. 


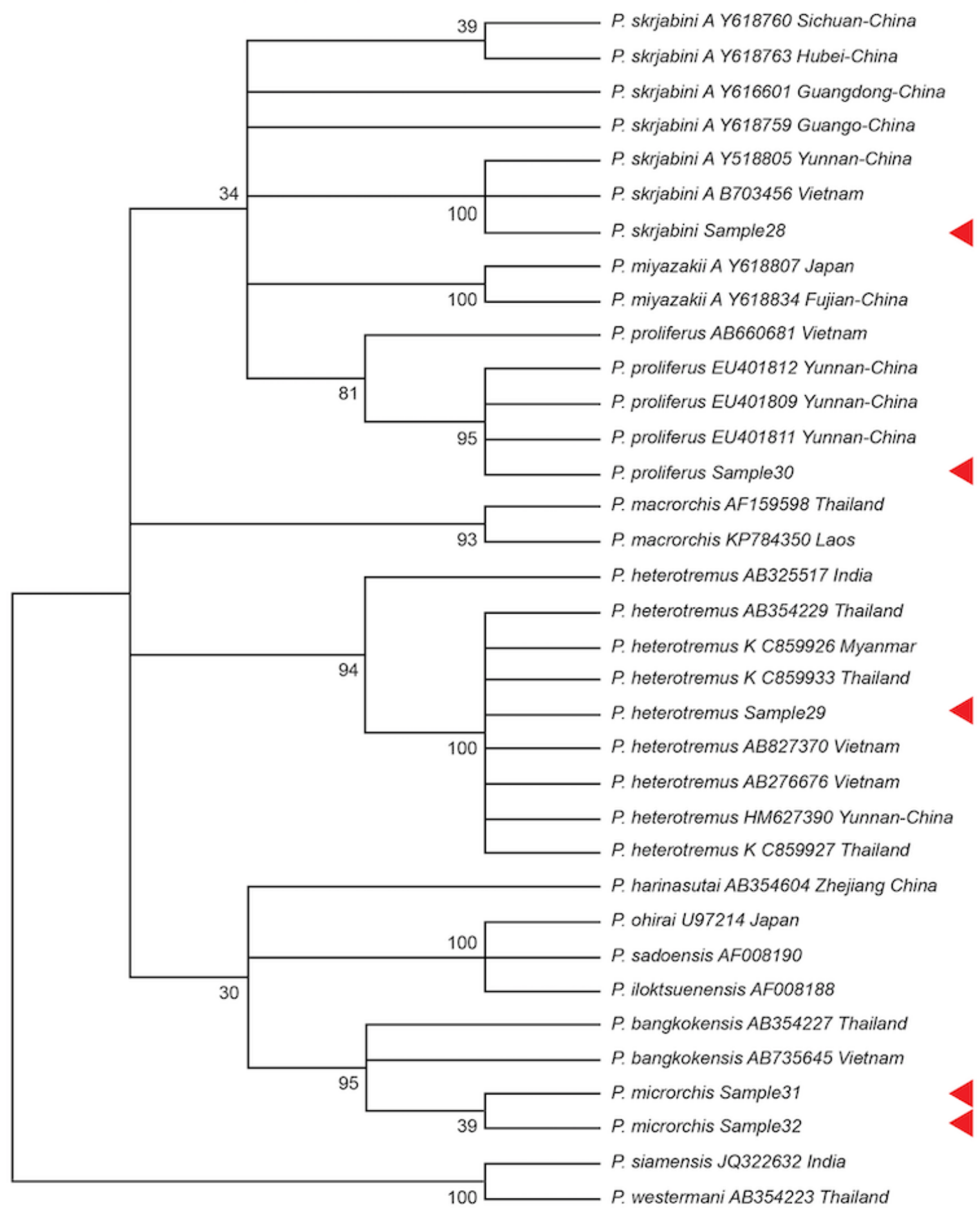

\section{Figure 2}

Phylogenetic tree by maximum parsimony method for $\mathrm{COI}$ gene. 


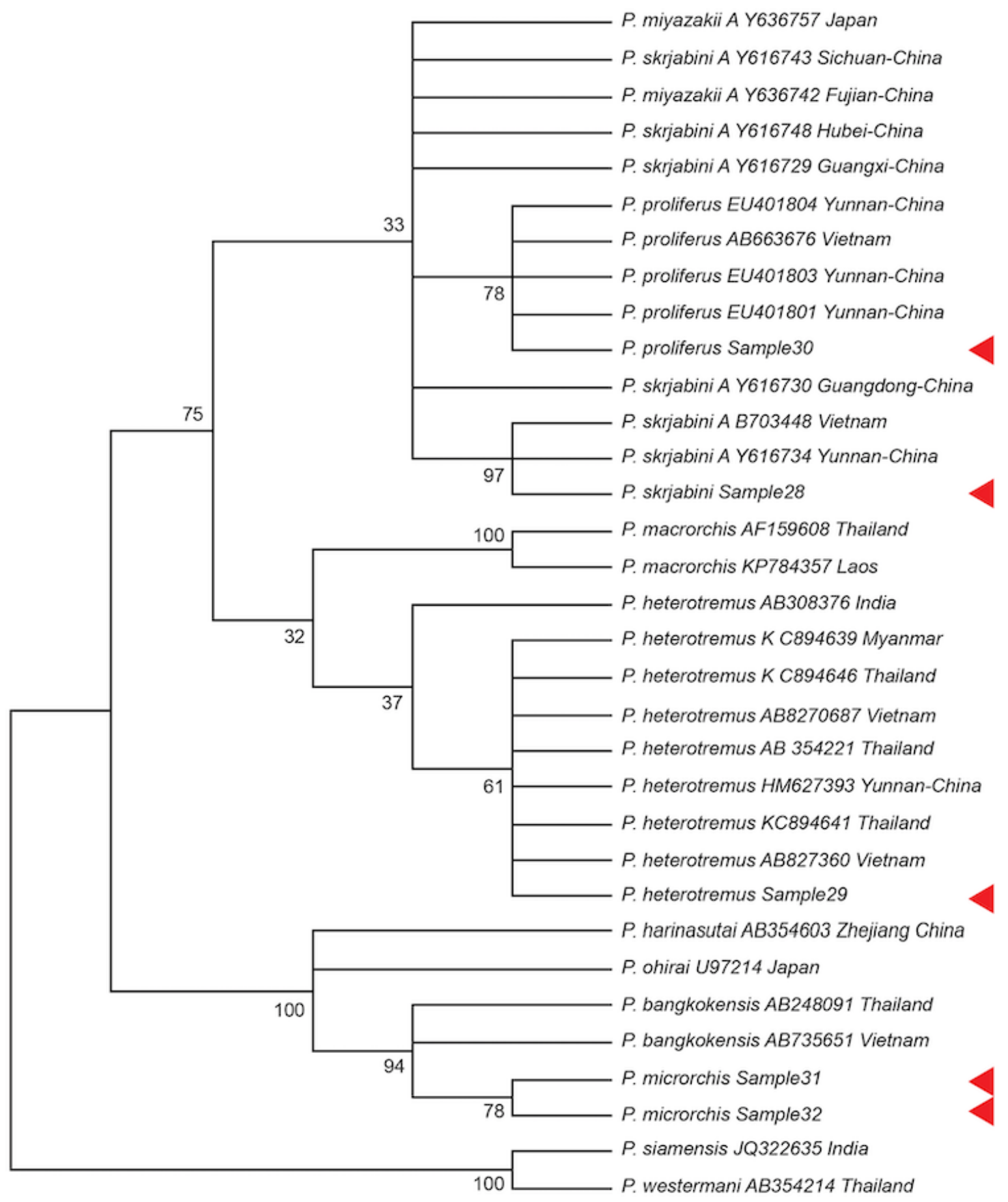

\section{Figure 3}

Phylogenetic tree by maximum parsimony method for ITS 2 gene. 


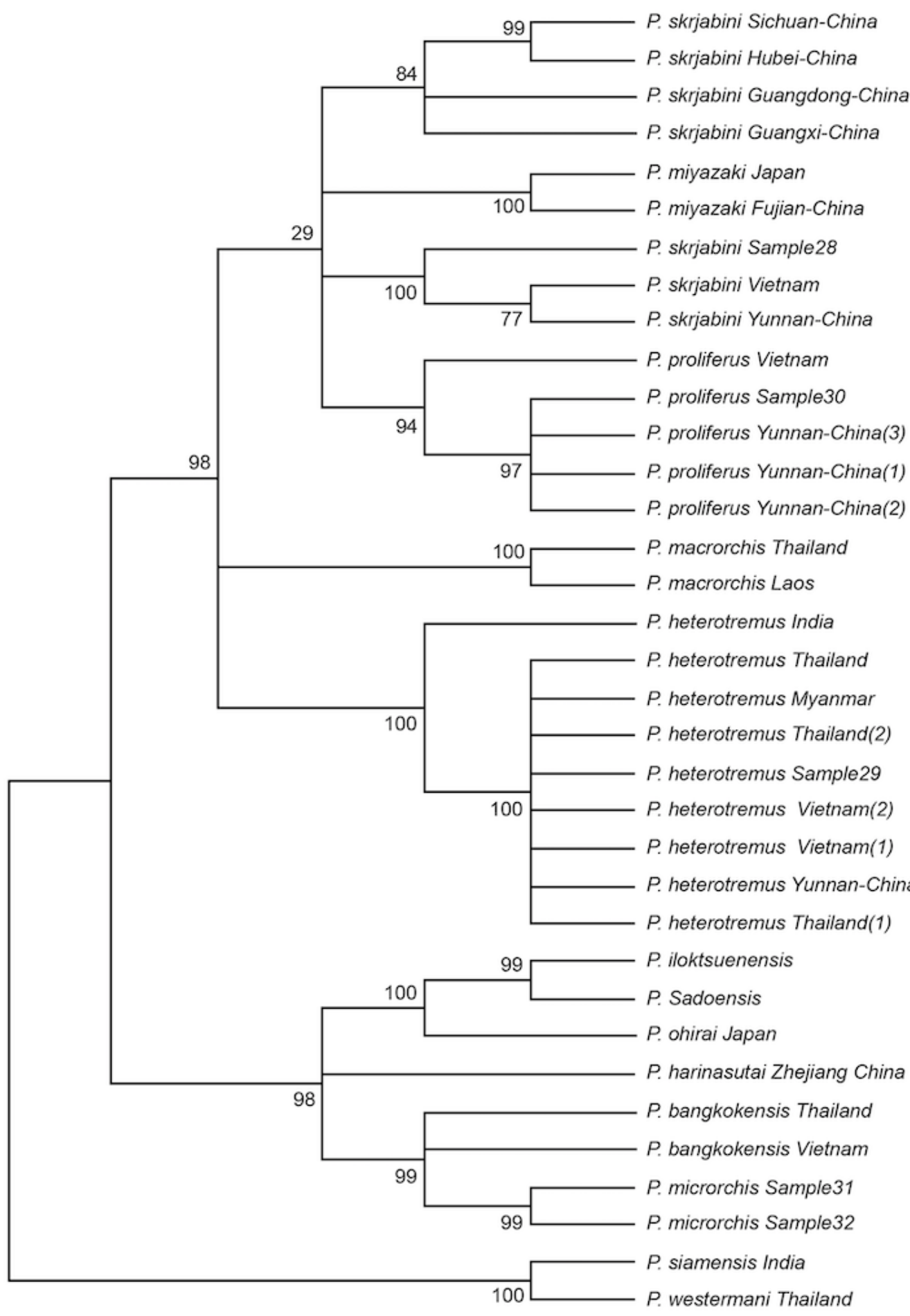

\section{Figure 4}

Phylogenetic tree by maximum parsimony method with both COI and ITS 2 regions.

\section{Supplementary Files}

This is a list of supplementary files associated with this preprint. Click to download. 
- Graphicalabstract.tif

Page 15/15 